\title{
Estructura de las esmeraldas de Muzo
}

\author{
Rev. Acad. Colomb. Cienc. Ex. Fis. Nat. 1953, 9 (33-34): 37-43.
}

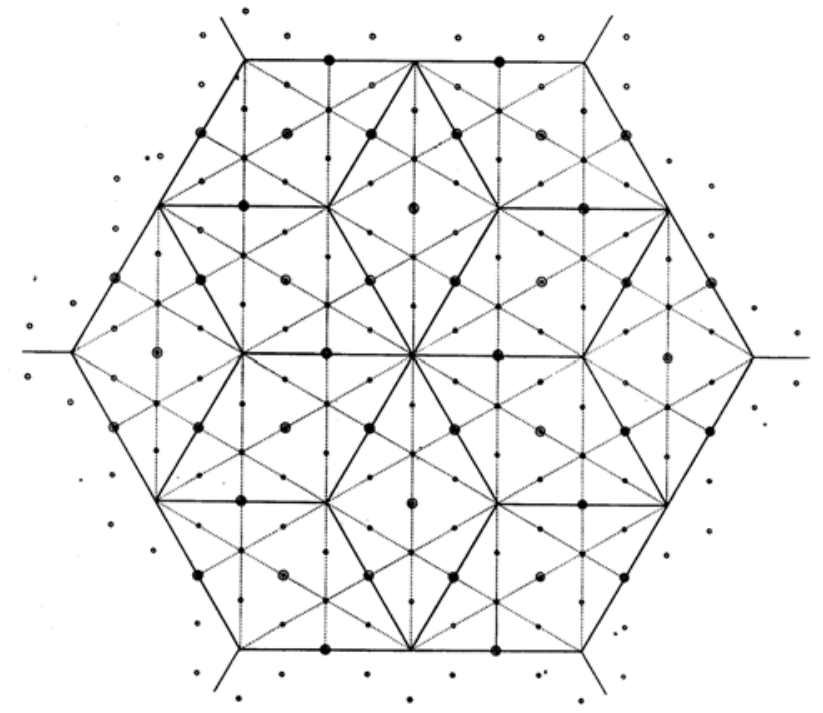

Posición de los átomos en la celda unidad, obtenidos a partir de los datos de difracción medidos.

\section{Antonio María Barriga Villalba (1894-1985)}

El multifacético científico Antonio María Barriga Villalba fue filósofo, lingüista, químico, físico, biólogo, investigador y humanista y director de la Casa de la Moneda de Bogotá. Autor de libros de Numismática, botánica, zoología. mineralogía y antropología. Investigó la composición de las aguas termales del país; el borrachero, el cacao sabanero; la circulación de la sangre en las alturas; especial mención merece su contribución al estudio de las esmeraldas de Colombia. $\mathrm{Su}$ actividad científica fue reconocida con: la Cruz de Boyacá; premio Javier Conde Barriga de Madrid; Miembro de la Real Academia de Ciencias de España; de la Sociedad de Cardiología (Fundador); de la Sociedad Colombiana de Física; de la Sociedad Colombiana de Medicina y Ciencias Naturales; de la Real Academia Hispanoamericana; de la Sociedad Numismática de Colombia y de la Academia Colombiana de Ciencias Exactas, Físicas y Naturales. Fue un entusiasta defensor del desarrollo científico del país.

www.encolombia.com/Antonio María Barriga Villalba, El Multifacético

Información suplementaria: http://www.accefyn.org.co/sp/ academicos/Silla_3_Antonio_Maria_Barriga_Villalba.htm
El estudio sobre la Estructura de las esmeraldas de Muzo, es un interesante trabajo que se considera como un clásico, por ser pionero en Colombia en el área de la cristalografía. El autor hace una descripción muy didáctica del fenómeno de la difracción de rayos $\mathrm{X}$, producida por materiales cristalinos. En la publicación se describe con detalle la determinación de la estructura: indexado, parámetros de la celda, el grupo espacial, número de moléculas en la celda unidad de una esmeralda de Muzo, a partir de los datos de difracción en una muestra policristalina y en monocristal. El autor revela un gran conocimiento de la cristalografía por difracción de rayos $\mathrm{X}$, describe con detalle la técnica y elabora de manera manual los cálculos. Los diagramas de difracción son de muy alta calidad. Pero el valor más importante del artículo es la interpretación de los resultados. Vale la pena destacar el cuidadoso procedimiento experimental empleado en la toma de datos, especialmente en el caso de las muestras monocristalinas, que a pesar de las irregularidades y defectos de las caras obtiene medidas muy precisas y constantes en todas las posiciones, lo cual le permite obtener una gran correspondencia entre los datos de la muestra policristalina con los del monocristal, para definir el grupo espacial de la esmeralda de Muzo y explicar sus dos formas comunes: la dextrorsum y la sinestrorsum, lo cual se resalta en la figura.

Estas razones ameritan considerar que este trabajo en cristalografía es una excelente realización para su época en Colombia, considerándose como el primer trabajo estructural en este campo.

Fernando Martínez Ortega, Docteur en Chimie Miembro Correspondiente

Edgar Páez Mozo, Ph. D., Chemistry Miembros de Número 


\section{ESTRULTURA DE LAS ESMERALDAS DE MUZD}

\section{A. M. BARRIGA VILLALBA}

Director de la Casa de Moneda
El estudio de la estructura fina de la materia por medio de los rayos $\mathrm{X}$, ha permitido establecer de manera definitiva que los cuerpos simples y compuestos están formados por átomos y no por moléculas, como lo habían establecido los químicos, átomos que ocupan posiciones determinadas, dentro de un retículo tridimensional, característico de cada sustancia. Al lado de las propiedades físicas y químicas de los cuerpos, es necesario hacer figurar el valor de las distancias interplanares por ser la más precisa definición de toda sustancia, cristalina o amorfa.

El ensayo que hoy nos permitimos presentar a los Honorables Académicos, tiene por objeto la medida del paralelipípedo elemental de la esmeralda de Muzo, y mostrar la forma de la red espacial que forman los átomos en este cristal, modelo de la más alta simetría.
Empleamos en las medidas del retículo el instrumento construido por North American Philips Company.

Consta el espectógrafo de difracción de rayos X.

I. Un generador de rayos $\mathrm{X}$.

II. Goniómetro analizador.

III. Un dispositivo electrónico, muy sensible, el cual por medio de un contador GeigerMuller, permite medir el Quantum de la radiación difractada por el espécimen.

IV. Un potenciómetro automático que inscribe la curva e intensidad correspondiente a las líneas del espectro entre $0^{\circ}$ y $45^{\circ}$.

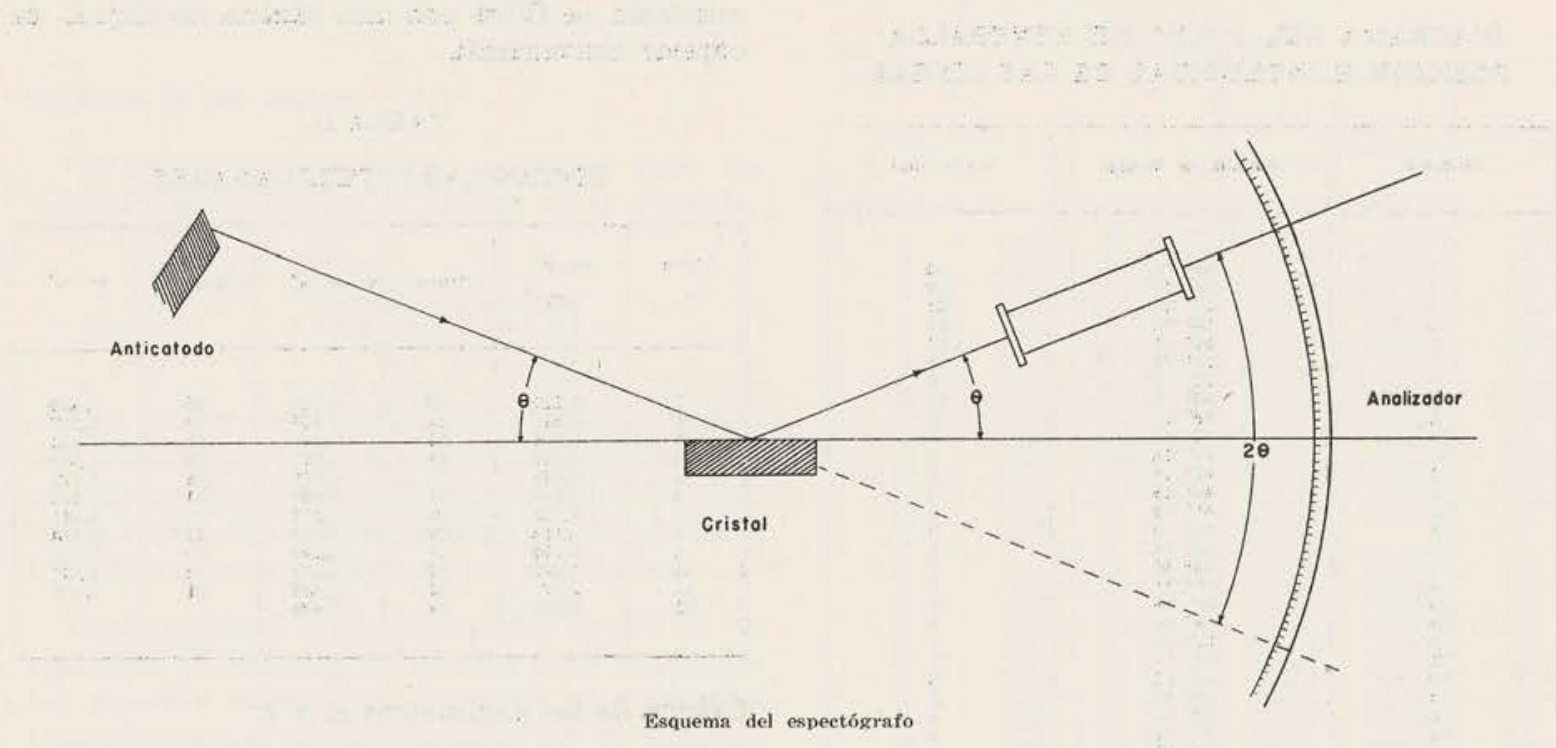

Las medidas pueden hacerse sobre el polvo de la sustancia o sobre cristales de la misma. En el primer caso, un gran número de cristalitos se encontrarán convenientemente orientados para difractar los rayos X. En el segundo, la cara de cristal, formada por mallas de átomos, producirá el fenómeno, exactamente como un rayo luminoso al incidir sobre una red, con la diferencia de que siendo tan pequeña la longitud de onda de los rayos $\mathrm{X}$ no puede ocurrir el fenómeno sino en una escala del orden del diámetro atómico, y se produce porque la radiación incidente origina el salto de un electrón de una órbita a otra, con la producción de energía radiante de la misma longitud de onda.
El fenómeno se sucede efectivamente como si fuera una simple difracción, auncuando es de un orden muchísimo más complejo que el de la luz.

El diagrama de difracción del polvo de esmeralda esta caracterizado por treinta y cinco líneas repartidas entre los $10^{\circ} .74$ y $87^{\circ}$. $43 \mathrm{La}$ intensidad es máxima a los $30^{\circ} 70$ y decrece, no de una manera regular, hasta los $90^{\circ}$ (Angulo de Bragg).

El polvo se preparó de esmeralda pura. Se pasó por tamiz de 200 mallas por pulgada, y se fijó simplemente por presión, sin aglutinante, sobre una lámina tallada en la forma usual para microscopia. 

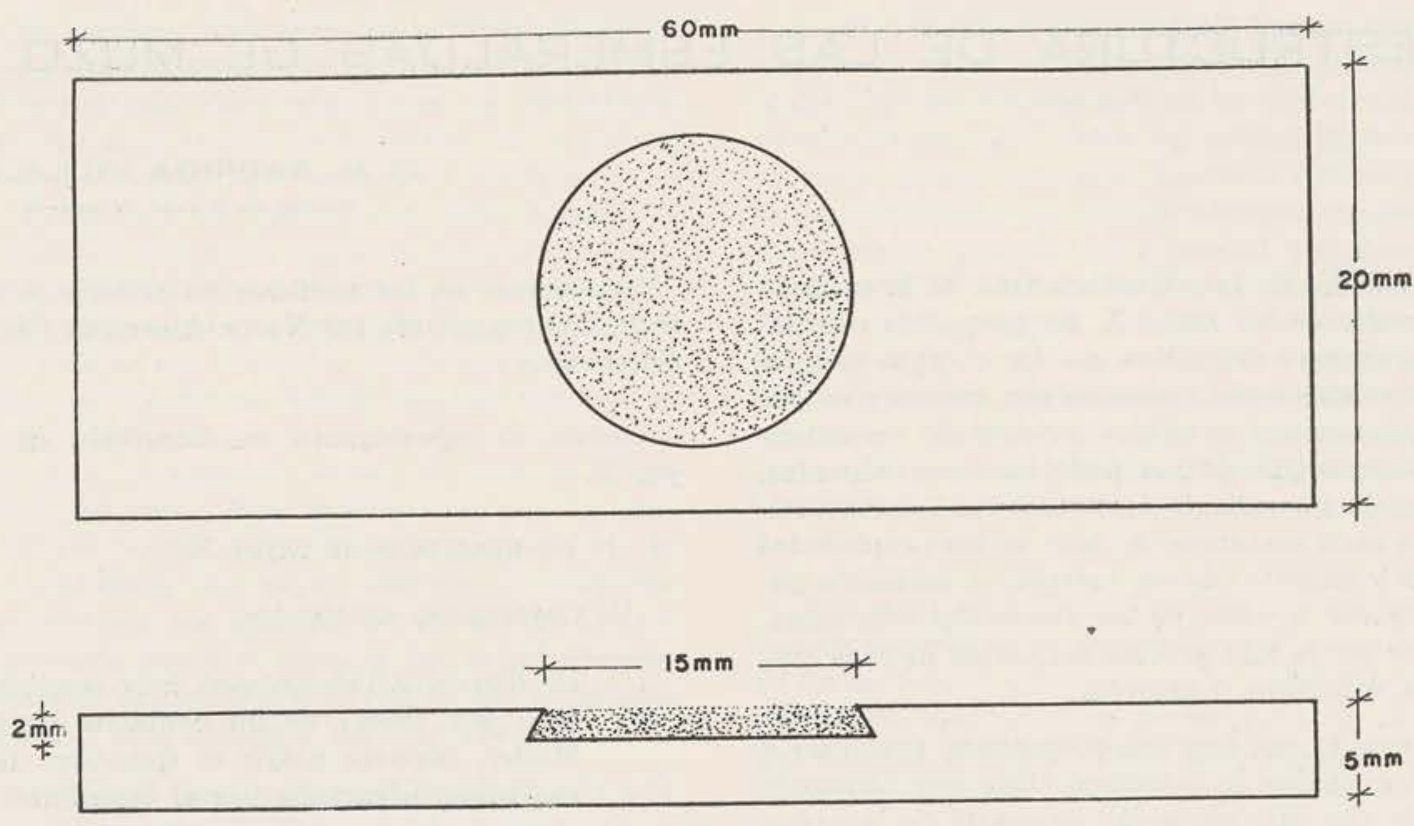

Portaobjeto pars polvo de esmeralda.

TABLA I

DIAGRAMA DEL POLVO DE ESMERALDA. POSICION E INTENSIDAD DE LAS LINEAS

\begin{tabular}{|c|c|c|}
\hline Número & Angulo de Bragg & Intensidad \\
\hline $\begin{array}{r}1 \\
2 \\
3 \\
4 \\
5 \\
6 \\
7 \\
7 \\
8 \\
9 \\
10 \\
11 \\
12 \\
13 \\
14 \\
15 \\
16 \\
17 \\
18 \\
19 \\
20 \\
21 \\
22 \\
23 \\
24 \\
25 \\
26 \\
27 \\
28 \\
29 \\
30 \\
31 \\
32 \\
33 \\
33 \\
35\end{array}$ & 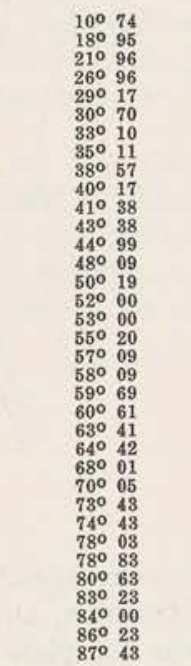 & $\begin{array}{r}43 \\
30 \\
25 \\
58 \\
52 \\
66 \\
6 \\
22 \\
5 \\
5 \\
4 \\
8 \\
3 \\
14 \\
2 \\
9 \\
13 \\
7 \\
9 \\
18 \\
5 \\
4 \\
21 \\
9 \\
8 \\
1 \\
2 \\
9 \\
8 \\
2 \\
7 \\
2 \\
2 \\
3 \\
2 \\
4 \\
1\end{array}$ \\
\hline
\end{tabular}

Las distancias interplanares correspondientes a cada una de las líneas del diagrama, se pudieron valorar por medio de la ecuación de Bragg:

$$
\mathrm{n} \lambda=2 \delta \operatorname{Sen} \theta(1)
$$

\footnotetext{
(1) $\delta=$ distancia interplanar.

$\mathrm{n}=$ orden de difracción.

$\lambda=$ longitud de onda correspondiente a la radiación del metal del anticátodo. (En este caso cobre).
}

Se empleó un tubo con anticátodo de cobre. La radiación se filtró con una lámina de níquel, de espesor conveniente.

TABLA II

DISTANCIAS INTERPLANARES

\begin{tabular}{|c|c|c|c|c|c|}
\hline $\begin{array}{c}\text { Orden } \\
(1)\end{array}$ & $\begin{array}{c}\text { en } \mathrm{A}^{0} \\
(2)\end{array}$ & Orden & en $\mathrm{A}^{0}$ & Orden & en $\mathrm{A}^{0}$ \\
& & & & & \\
\hline & & & & & \\
\hline 1 & & & & & \\
2 & 8,233 & 18 & 2,014 & 25 & 1,445 \\
3 & 4,681 & 14 & 1,859 & 26 & 1,377 \\
4 & 3,045 & 15 & 1,816 & 27 & 1,335 \\
5 & 3,060 & 16 & 1,757 & 28 & 1,288 \\
6 & 2,911 & 17 & 1,727 & 29 & 1,271 \\
7 & 2,705 & 19 & 1,663 & 30 & 1,213 \\
8 & 2,554 & 20 & 1,612 & 31 & 1,191 \\
9 & 2,333 & 21 & 1,587 & 32 & 1,160 \\
10 & 2,243 & 22 & 1,528 & 33 & 1,151 \\
11 & 2,181 & 23 & 1,466 & 35 & 1,127 \\
12 & 2,058 & 24 & 1,445 & & 1,115 \\
& & & & & \\
\hline
\end{tabular}

Cálculo de los parámetros a. y $c$.

Como más adelante lo demostraremos, con los monocristales de Muzo, las líneas primera y segunda del diagrama del polvo de esmeralda corresponden, respectivamente, a los planos 100 y 001 del prisma elemental.

La magnitud $a$. se puede deducir así:

$$
a=\frac{\delta 100}{\operatorname{Sen} 60^{\circ}}=9,5077
$$

y el valor del eje $c$. según la tabla I, es:

$$
2 \theta=18^{\circ}, 85=18^{\circ}, 57^{\prime} 0^{\prime \prime}
$$

(1) Ordenes de las lineas principales.

(2) $1 . \mathrm{A}^{\circ}=10^{-8} \mathrm{cms}$ 
y según la ley de Bragg ya citada, se tiene:

$$
c=\delta 001=\frac{\mathrm{A}^{\circ}}{1,5412}=\frac{\mathrm{A}^{\circ}}{2 \operatorname{Sen} 9^{\circ} .28,30^{\prime \prime}}=4,681 .
$$

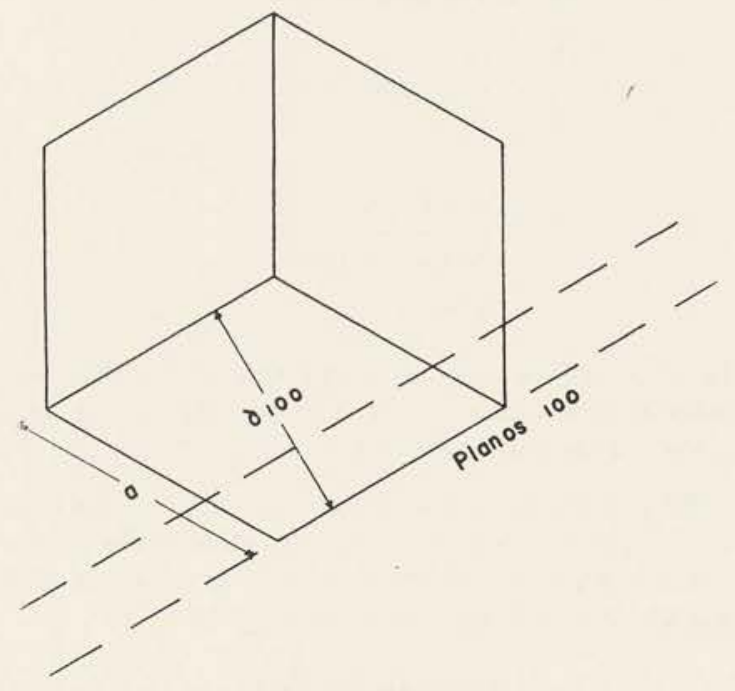

Cálculos de los índices.

Por sustitución de valores sucesivos para los índices $h . k$. $l$. (Indices de Miller) en la ecuación,

$$
\operatorname{Sen}^{2} \theta=\frac{\lambda^{2}}{4} \frac{4}{3}\left[\left(\frac{h^{2}+k^{2}+l k}{a^{2}}\right)+\frac{1^{2}}{c^{2}}\right]
$$

con los valores encontrados para a. y $c$. se pueden deducir los índices correspondientes a cada una de las líneas del diagrama de difracción del polvo y con ellos se comprueban si corresponden los valores encontrados con los calculados.

Pueden deducirse los índices por medio de las cartas de Hull-Davey calculadas para los distintos sistemas cristalinos, conocidas las distancias interplanares, o en nuestro caso por el valor de la relación de los ejes cristalinos tomándolo en la ordenada correspondiente, lo cual nos dará las distintas curvas cuyas características son las del plano reticular.

TABLA III

INDICES DEDUCIDOS CON EL VALOR C/A

\begin{tabular}{|ll|ll|ll|}
\hline 1. & 100 & 10. & 230 & 19. & 150 \\
2. & 110 & 11. & 131 & 20. & 250 \\
3. & 001 & 12. & 112 & 21. & 350 \\
4. & 101 & 13. & 140 & 22. & 160 \\
5. & 111 & 14. & 231 & 23. & 450 \\
6. & 201 & 15. & 122 & 24. & 232 \\
7. & 121 & 16. & 140 & 25. & 103 \\
8. & 130 & 17. & 120 & 26. & 203 \\
9. & 301 & 18. & 340 & & \\
\hline
\end{tabular}

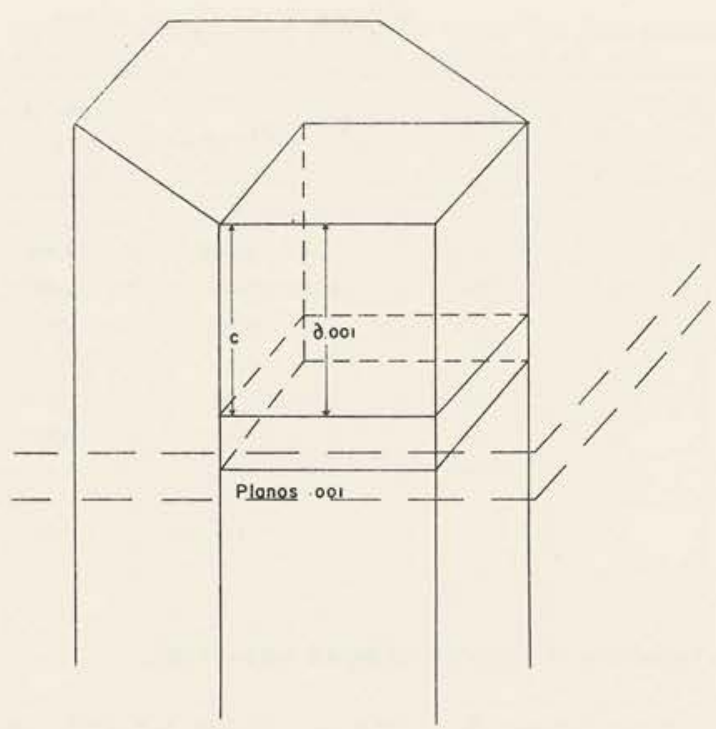

La Tabla IV, muestra la correspondencia entre los valores de $\operatorname{Sen}^{2} \theta$ calculados y experimentales, según los índices deducidos, exactos para las líneas principales que dan el valor del retículo y muy aceptables para las demás.

\begin{tabular}{|c|c|c|c|c|c|}
\hline Línea & $2 \theta$ & $\theta$ & $\begin{array}{l}\operatorname{sen}^{2} \theta \\
\text { Calculado }\end{array}$ & $\begin{array}{c}\operatorname{Sen}^{2} \theta \\
\text { Experimen- } \\
\text { tal }\end{array}$ & h k I \\
\hline 1. & $10^{\circ} 74$ & 5022,12 " & 0,008752 & 0,008758 & 100 \\
\hline 2 & 18095 & $9028,30^{\prime \prime}$ & 0,02710 & $0,02709^{\circ}$ & 001 \\
\hline 3 & 21096 & $10^{\circ} 58^{\circ} 48^{\prime \prime \prime}$ & 0,03502 & 0,03627 & 200 \\
\hline 4 & $26^{\circ} 96$ & $13028^{\prime}, 48^{\prime \prime \prime}$ & 0,05336 & 0,05433 & 111 \\
\hline 5 & 29017 & $14^{\circ} 35,6$ " & 0,06129 & 0,06341 & 120 \\
\hline 6 & 30070 & $15^{\circ} 21^{\prime}, 0$ " & 0,07880 & 0,07007 & 300 \\
\hline 7 & 33010 & $16^{\circ} 33^{\prime} \quad 0^{\prime \prime}$ & 0,07856 & 0,08113 & 301 \\
\hline 8 & 35011 & $17^{\circ} 33^{\prime}, 18^{\prime \prime}$ & 0,08839 & 0,09076 & 121 \\
\hline 9 & 38057 & 19017,6 " & 0,10507 & 0,10976 & 220 \\
\hline 10 & $40^{\circ} 17$ & $20^{\circ} 5, \quad 6 "$ & 0,11715 & $\begin{array}{l}0,11793 \\
0,11793\end{array}$ & 102 \\
\hline 11 & 41038 & $20^{\circ} 41^{\prime}, 24^{\prime \prime}$ & 0,12258 & $\begin{array}{l}0,12482 \\
0,1\end{array}$ & 131 \\
\hline 12 & $\begin{array}{l}40^{\circ} 38 \\
\end{array}$ & $21^{\circ} 41,24$, & 0,13466 & $\begin{array}{l}0,12482 \\
0,13659\end{array}$ & $\begin{array}{l}181 \\
112\end{array}$ \\
\hline 13 & $\begin{array}{l}40099 \\
44090\end{array}$ & $22029,42^{\prime}$, & 0,14342 & 0,14638 & 202 \\
\hline 14 & $\begin{array}{l}48099 \\
480\end{array}$ & $24^{\circ} 29^{\prime} 52^{\prime \prime}$ & 0,17190 & $\begin{array}{l}0,16969 \\
0,1699\end{array}$ & 212 \\
\hline 15 & 50019 & $25^{\circ} 5,42^{\prime \prime}$ & 0,18387 & 0,17987 & 140 \\
\hline 16 & 52000 & $26^{\circ} 0^{\prime}, 0$ " & 0,19346 & 0,19216 & 231 \\
\hline 17 & 53000 & $26^{\circ} \quad 30^{\prime} \quad 0^{\prime \prime}$ & 0,19909 & & \\
\hline 18 & & $27^{\circ} 36^{\prime} \quad 0^{\prime \prime}$ & 0,21347 & 0,21464 & 222 \\
\hline 19 & $57^{\circ} 09$ & $28^{\circ} 32^{\prime} 42^{\prime \prime}$ & 0,22223 & 0,22834 & 132 \\
\hline 20 & 58009 & $29^{\circ} \quad 2,42 "$ & 0,23641 & 0,23570 & 330 \\
\hline 21 & 59069 & $29^{\circ} 50^{\prime} \quad 42^{\prime \prime}$ & 0,24391 & 0,24766 & 003 \\
\hline 22 & $60 \% 1$ & $30^{\circ} 18^{\prime} \quad 18^{\prime \prime}$ & 0,25266 & 0,25402 & 103 \\
\hline 23 & $63^{\circ} 41$ & 31042,18 " & 0,27893 & 0,27619 & 203 \\
\hline 24 & 54042 & 32012,36 " & 0,28270 & 0.28411 & 151 \\
\hline 25 & 68001 & $340^{\circ} 0,18$ " & 0,31522 & 0,31277 & 600 \\
\hline 26 & 70005 & $\begin{array}{llll}35^{\circ} & 15 & 0\end{array}$ & 0,33290 & 0,32730 & $\begin{array}{l}600 \\
502\end{array}$ \\
\hline 27 & 73043 & $36^{\circ} 42^{\prime}, 54 "$ & 0,35774 & 0,35740 & $\begin{array}{l}\text { Doz } \\
313\end{array}$ \\
\hline 28 & 74043 & $37 \circ 1^{\prime}, 54 "$ & 0,36859 & 0,36579 & 521 \\
\hline 29 & $780^{\circ} 03$ & $39^{\circ} 0$, 54" & 0,39624 & & \\
\hline 30 & 78083 & $39^{\circ} 24^{\prime} 54^{\prime \prime}$ & 0,41020 & 0,40314 & 323 \\
\hline 31 & $80^{\circ} 63$ & $40^{\circ} 18^{\prime} 54^{\prime \prime}$ & 0,41858 & 0,42030 & 440 \\
\hline 32 & $83^{\circ} 23$ & $41^{\circ} 36^{\prime} 54^{\prime \prime}$ & 0,44237 & 0,44105 & 104 \\
\hline 33 & 84000 & $42^{\circ} \quad 0^{\prime} \quad 0^{\prime \prime}$ & 0,44769 & 0,44739 & 441 \\
\hline 34 & $86^{\circ} 23$ & $43^{\circ} \quad 6^{\prime} 54^{\prime \prime}$ & 0,46870 & 0,46712 & 204 \\
\hline 35 & 87043 & $43^{\circ} 42,54 "$ & 0,47759 & & \\
\hline
\end{tabular}

TABLA IV

Para el sistema exagonal, la distancia interplanar tiene por expresión:

$$
\delta=\frac{a}{\sqrt{\frac{4}{3}\left(h^{2}+k^{2}+h k\right) \frac{l^{2} a^{2}}{c^{2}}}}
$$

que sirve para el cálculo de $a$ en las reflexiones h. k. l. Según los datos de la Tabla IV, podemos poner: 
TABLA V

\begin{tabular}{|c|c|c|c|}
\hline Ordenes & Indices & $\delta \sqrt{\frac{4}{3}-\left(h^{2}+k^{2}+h k\right.}$. & $\begin{array}{l}\text { Valor de } \\
\qquad a\end{array}$ \\
\hline 1 & 100 & $2,233 \times 1,1546$ & 9,505 \\
\hline 3 & 200 & $4,045 \times 2,30$ & 9,303 \\
\hline 5 & 120 & $3,060 \times 3,05$ & 9,330 \\
\hline 6 & 300 & $2,911 \times 3,46$ & 10,072 \\
\hline 9 & 220 & $2,333 \times 4,00$ & 9,332 \\
\hline 15 & 140 & $1,816 \times 5,29$ & 9,606 \\
\hline 25 & 600 & $1,377 \times 6,92$ & 9,588 \\
\hline & & Promedio & 8,525 \\
\hline
\end{tabular}

\section{Volumen del paralelipípedo elemental.}

El volumen de la malla unidad, teniendo presentes los valores encontrados para los ejes $a$. y $c$. será:

$$
\begin{aligned}
& V=a^{2} c \cdot \operatorname{Sen} 60^{\circ} \\
& V=366,456
\end{aligned}
$$

$\mathrm{A}^{\circ}$ cúbicos. Puede también expresarse en centímetros cúbicos.
Número de moléculas en la célula unidad.

Designamos por:

$\mathrm{N}=$ número de moléculas

$\mathbf{M}=$ Peso molecular

$\mathrm{d}=$ Densidad media

$\mathrm{V}=$ Volumen en $\mathrm{A}^{\mathrm{O}}$

Tendremos :

$$
\begin{aligned}
& \mathrm{N}=\frac{\mathrm{V} \cdot \mathrm{d} \cdot 6,06 \times 10^{23}}{\mathrm{M}}=\frac{\mathrm{V} \cdot \mathrm{d} .}{\mathrm{M} \times 1,65} \\
& \mathrm{~N}=\frac{366,456 \times 2,66}{537,36 \times 1,65} \\
& \mathrm{~N}=1,098
\end{aligned}
$$

Lo cual indica que en el prisma elemental hay solamente los átomos correspondientes a una sola molécula de esmeralda $\left(\mathrm{Be}_{3} \mathrm{Al}_{2} \mathrm{Si}_{6} 0_{18}\right)$.

El prisma exagonal de la piedra preciosa contiene tres veces el número de átomos de la expresión anterior, de manera que la fórmula química y mineralógica de la gema será:

$$
\left(\begin{array}{llll}
\mathrm{Be}_{3} & \mathrm{Al}_{2} & \mathrm{Si}_{6} & 0_{18}
\end{array}\right)^{3} \text {. }
$$

Como se puede observar, el diagrama de difracción del polvo de esmeralda sirve para me-

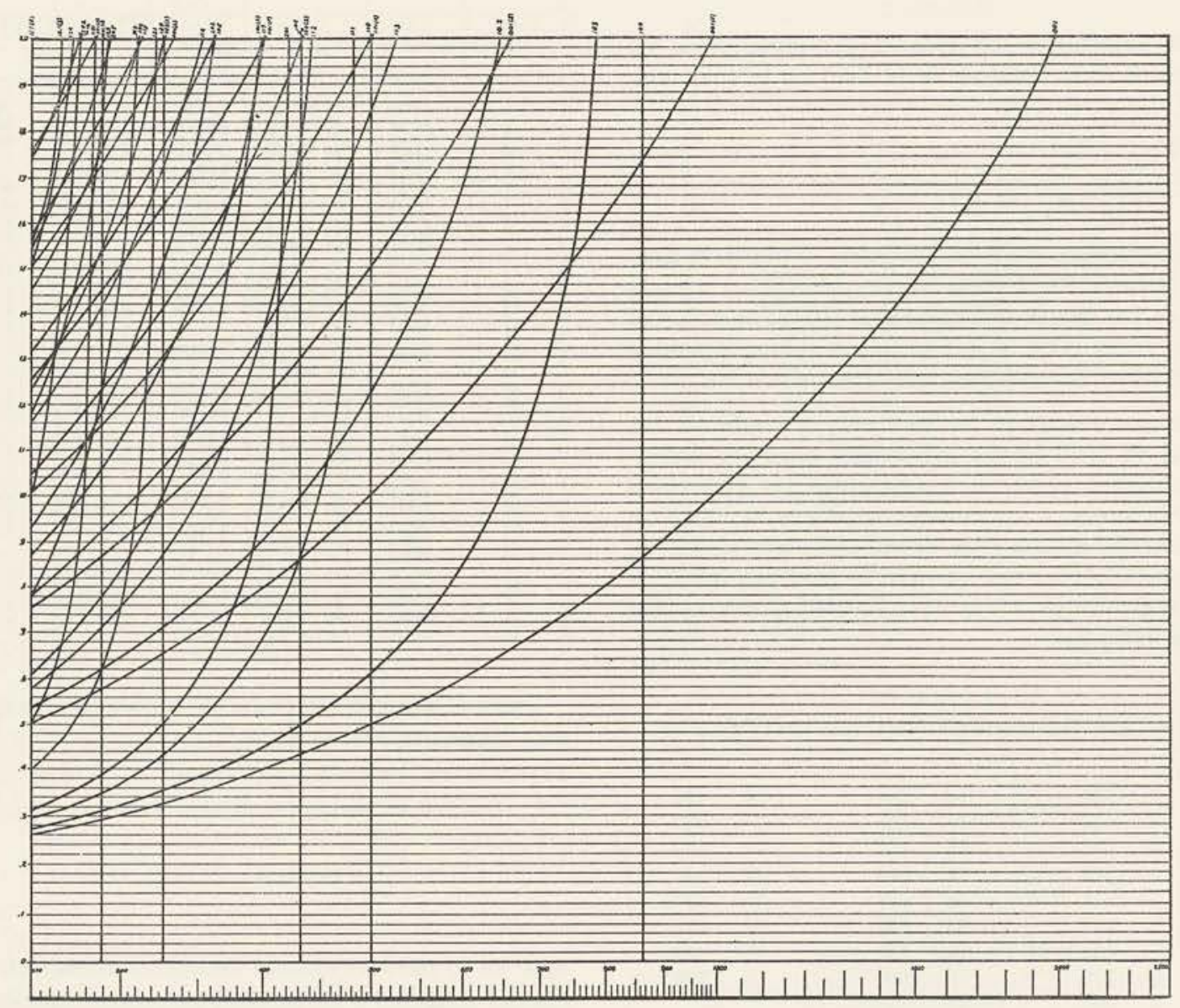

Carta de Hull-Davey. 
dir exactamente los dos ejes cristalográficos $a$. y $c$. del prisma romboidal elemental de la singonia exagonal, en el caso de la esmeralda, conocida la posición de las dos primeras líneas del diagrama.

\section{Diagramas con monocristales de esmeralda.}

Los diagramas se obtuvieron con esmeraldas de más de 20 quilates, bien formadas, haciendo incidir la radiación sobre la cara 001 del cristal, sin ningún pulimento, en rotaciones sucesivas de $30^{\circ}$ en $30^{\circ}$ alrededor del punto medio. Lo mismo se practicó para las caras laterales. Ha sido sorprendente el resultado tan constante obtenido en todas las posiciones, a pesar de las irregularidades y defectos de las caras. Las tablas VI y VII muestran los valores comunes tabulados según el ángulo de Bragg.

TABLA VI

VALORES DE $2 \theta$ EN LA BASE DEL CRISTAL

\begin{tabular}{|c|c|c|c|c|c|c|c|c|c|c|c|c|c|c|}
\hline $\begin{array}{c}\text { Rotación } \\
0^{\circ} \\
30^{\circ} \\
60^{\circ} \\
90^{\circ} \\
120^{\circ} \\
150^{\circ} \\
180^{\circ}\end{array}$ & $\begin{array}{l}19^{\circ} 02 \\
19^{\circ} 05 \\
19^{\circ} 29 \\
19^{\circ} 40 \\
19^{\circ} 45 \\
19^{\circ} 09 \\
19^{\circ} 25\end{array}$ & $\begin{array}{l}26^{\circ} 26 \\
26^{\circ} 16\end{array}$ & $27 \circ 87$ & $28^{\circ} 67$ & $\begin{array}{l}34098 \\
34090 \\
34098 \\
34088 \\
34084 \\
34088 \\
34088\end{array}$ & $\begin{array}{l}38058 \\
38058 \\
38058 \\
38058 \\
38058 \\
38058 \\
38058\end{array}$ & $\begin{array}{l}39^{\circ} 12 \\
390^{\circ} 08 \\
39^{\circ} 06 \\
39^{\circ} 06 \\
38098 \\
38^{\circ} 71 \\
38^{\circ} 71\end{array}$ & $49^{\circ} 19$ & $\begin{array}{l}53^{\circ} 78 \\
53^{\circ} 54 \\
533^{\circ} 65 \\
53^{\circ} 53 \\
53^{\circ} 53 \\
53^{\circ} 50 \\
53^{\circ} 48\end{array}$ & $\begin{array}{l}59^{\circ} 61 \\
59^{\circ} 55 \\
59054 \\
590_{44} \\
59^{\circ} 54 \\
59^{\circ} 61 \\
59^{\circ} 61\end{array}$ & $\begin{array}{l}60^{\circ} 35 \\
600^{\circ} 21 \\
600_{21}^{\circ} \\
600^{\circ} 21 \\
60^{\circ} 21 \\
60^{\circ} 21 \\
60^{\circ} 09\end{array}$ & $\begin{array}{l}74 \circ 03 \\
73 \circ 97 \\
73 \circ 96 \\
73097 \\
73 \circ 96 \\
73093 \\
73093\end{array}$ & $\begin{array}{l}83^{\circ} 21 \\
83^{\circ} 24 \\
83^{\circ} 24 \\
83^{\circ} 17 \\
83^{\circ} 31 \\
83^{\circ} 25 \\
83^{\circ} 25\end{array}$ & $\begin{array}{l}84004 \\
83092 \\
83092 \\
83^{\circ} 84 \\
833^{\circ 97} \\
830^{\circ} \\
83^{\circ} 44\end{array}$ \\
\hline & $19^{\circ} 22$ & & & & $34^{\circ} 90$ & $38^{\circ} 58$ & $38 \circ 96$ & & $53^{\circ} 57$ & $59^{\circ} 55$ & $60^{\circ} 21$ & $73^{\circ 96}$ & $83^{\circ} 23$ & $83^{\circ} 80$ \\
\hline
\end{tabular}

TABLA VII

VALORES DE 2 f EN LAS CARAS LATERALES

\begin{tabular}{|c|c|c|c|c|c|c|c|c|c|c|}
\hline Planos & & & & & & & & & & \\
\hline $\begin{array}{l}100 \\
100 \\
010 \\
010 \\
100 \\
100\end{array}$ & $\begin{array}{l}10^{\circ} 56 \\
10^{\circ} 54 \\
10^{\circ} 68 \\
10^{\circ} 54 \\
10^{\circ} 54 \\
10^{\circ} 54\end{array}$ & $\begin{array}{l}11^{\circ} 02 \\
10^{\circ 94} \\
10^{\circ} 94\end{array}$ & $\begin{array}{l}21089 \\
21084 \\
20096 \\
21^{\circ} 76 \\
21066 \\
21076\end{array}$ & $\begin{array}{l}32^{\circ 997} \\
33^{\circ} 11 \\
32^{\circ} 97 \\
33^{\circ} 11\end{array}$ & $\begin{array}{l}33^{\circ} 37 \\
33^{\circ} 17 \\
33 \% 51 \\
33051 \\
33^{\circ} 37\end{array}$ & $\begin{array}{l}44099 \\
44079 \\
44059 \\
44067 \\
44059 \\
44099\end{array}$ & $\begin{array}{l}56^{\circ} 90 \\
56^{\circ} 70 \\
56^{\circ} 90 \\
56^{\circ} 76 \\
56^{\circ} 80 \\
57^{\circ} 01\end{array}$ & $\begin{array}{l}57^{\circ} 57 \\
57^{\circ} 44 \\
57^{\circ} 31 \\
57^{\circ} 36 \\
57^{\circ} 41 \\
57^{\circ} 41\end{array}$ & $\begin{array}{l}70052 \\
700^{\circ} \\
70^{\circ} 12 \\
700^{\circ} \\
700^{\circ} \\
700_{09}\end{array}$ & $\begin{array}{l}84.24 \\
84.24 \\
84.24 \\
84^{\circ} 0_{14} \\
84^{\circ} 14 \\
84^{\circ} 24\end{array}$ \\
\hline & $10^{\circ} 50$ & $10^{\circ} 96$ & $21^{\circ} 64$ & $33^{\circ} 04$ & $33^{\circ} 38$ & $44^{\circ} 77$ & 56084 & $57^{\circ} 41$ & $70^{\circ} 26$ & $84^{\circ} 20$ \\
\hline
\end{tabular}

Utilizando los datos de estas dos Tablas correspondientes a las caras del cristal, construimos la
Tabla VIII que prueba la concordancia de las medidas.

TABLA VIII

\begin{tabular}{|c|c|c|c|c|c|c|c|}
\hline Caras & Indices & d.en $A^{\circ}$ & $2 \theta$ & $\theta$ & $\theta^{\circ}$ & $\begin{array}{c}\operatorname{Sen}^{2} \\
\text { Experimental }\end{array}$ & $\begin{array}{c}\operatorname{Sen}^{2} \\
\text { Calculado }\end{array}$ \\
\hline $\begin{array}{l}1 \mathrm{C} . \\
1 \mathrm{~B} . \\
2 \mathrm{C} . \\
3 \mathrm{C} . \\
2 \mathrm{~B} . \\
4 \mathrm{C} . \\
5 \mathrm{C} . \\
3 \mathrm{~B} . \\
6 \mathrm{C} . \\
4 \mathrm{~B} \\
7 \mathrm{C}\end{array}$ & $\begin{array}{l}100 \\
001 \\
200 \\
300 \\
220 \\
202 \\
132 \\
103 \\
502 \\
104 \\
441\end{array}$ & $\begin{array}{l}8,248 \\
4,614 \\
4,104 \\
2,744 \\
2,332 \\
2,023 \\
11,611 \\
1,536 \\
1,339 \\
1,160 \\
1,149\end{array}$ & $\begin{array}{l}10073 \\
19022 \\
21064 \\
33021 \\
38058 \\
44077 \\
57012 \\
60021 \\
70026 \\
83023 \\
84020\end{array}$ & 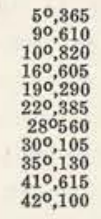 & 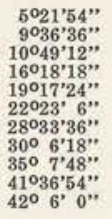 & $\begin{array}{l}0,008736 \\
0.027882 \\
0,035253 \\
0.072826 \\
0.10111 \\
0,145030 \\
0,228560 \\
0,255692 \\
0,331131 \\
0.441002 \\
0,449436\end{array}$ & $\begin{array}{l}0,008752 \\
0,027104 \\
0,034993 \\
0,07809 \\
0,105090 \\
0,140100 \\
0,222230 \\
0,250482 \\
0,327300 \\
0.440193 \\
0,447397\end{array}$ \\
\hline
\end{tabular}

$\mathrm{C}=$ caras laterales. $\mathrm{B}=$ base de cristal.

A pesar de los errores cometidos y de las irregularidades que siempre presentan en su superficie los cristales naturales es plenamente satisfactoria la concordancia en las cifras experimentales y calculadas para $\operatorname{Sen}^{2} \theta$. De las 17 líneas del diagrama de la base y del 23 de las caras laterales, hemos seleccionado aquellas comunes y más homogéneas que figuran en todos los diagramas y que forman los tripletes y dobletes característicos.

Comparando los diagramas de difracción del polvo de esmeralda y los de los cristales se observa una correspondencia aceptable, como puede verse en la Tabla IX.
TABLA IX

\begin{tabular}{|c|c|c|}
\hline $\begin{array}{c}\text { Diagrama } \\
\text { del polvo } \\
2 \theta\end{array}$ & $\begin{array}{c}\text { Diagrama } \\
\text { de las caras laterales } \\
29\end{array}$ & $\begin{array}{c}\text { Diagrama } \\
\text { de la base } \\
2 \theta\end{array}$ \\
\hline 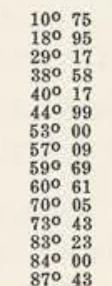 & 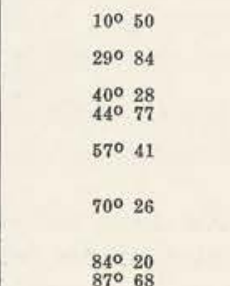 & $\begin{array}{l}19^{\circ} 22 \\
38^{\circ} 57 \\
53^{\circ} \quad 57 \\
59^{\circ} \quad 55 \\
60^{\circ} 21 \\
73^{\circ} \quad 96 \\
83^{\circ} 23\end{array}$ \\
\hline
\end{tabular}




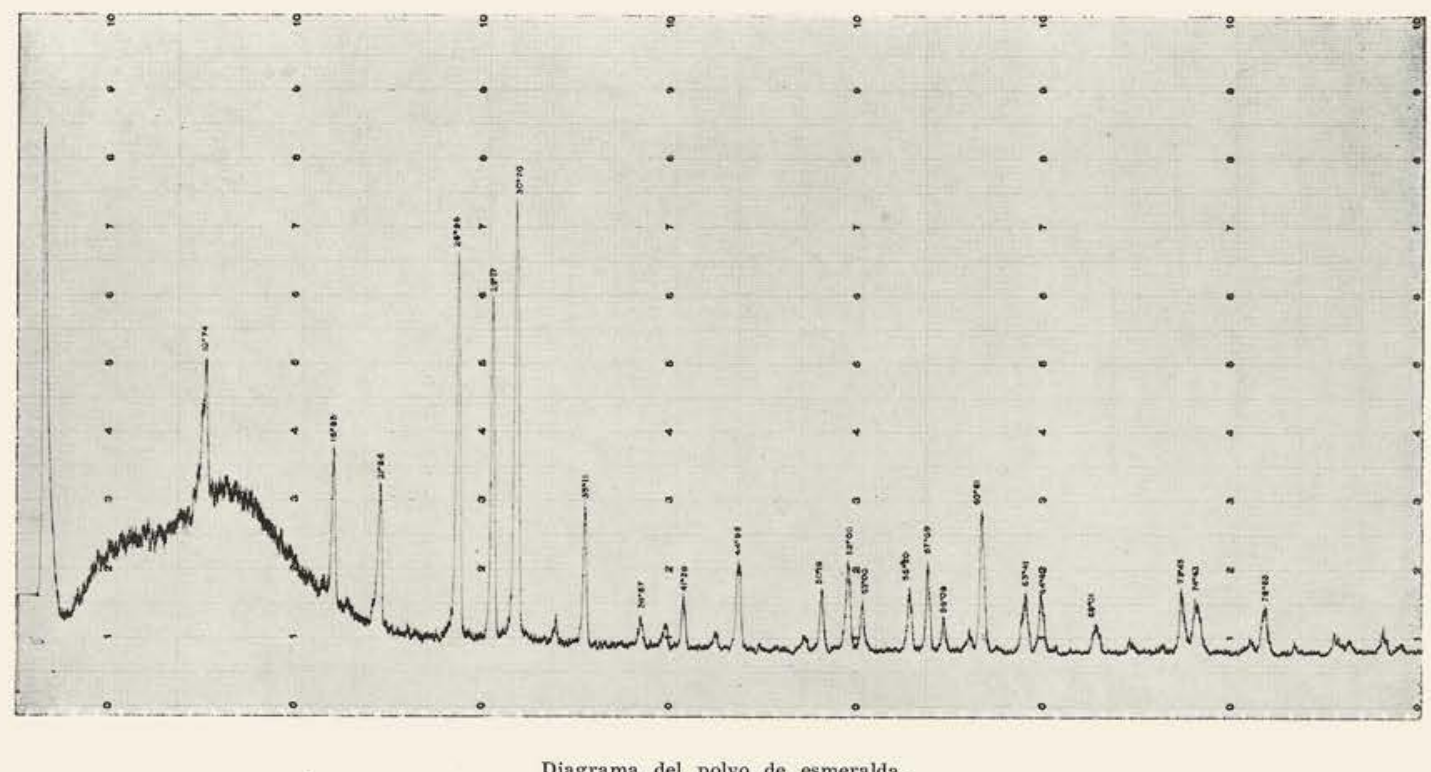

Diagrama del polvo de esmeralda.

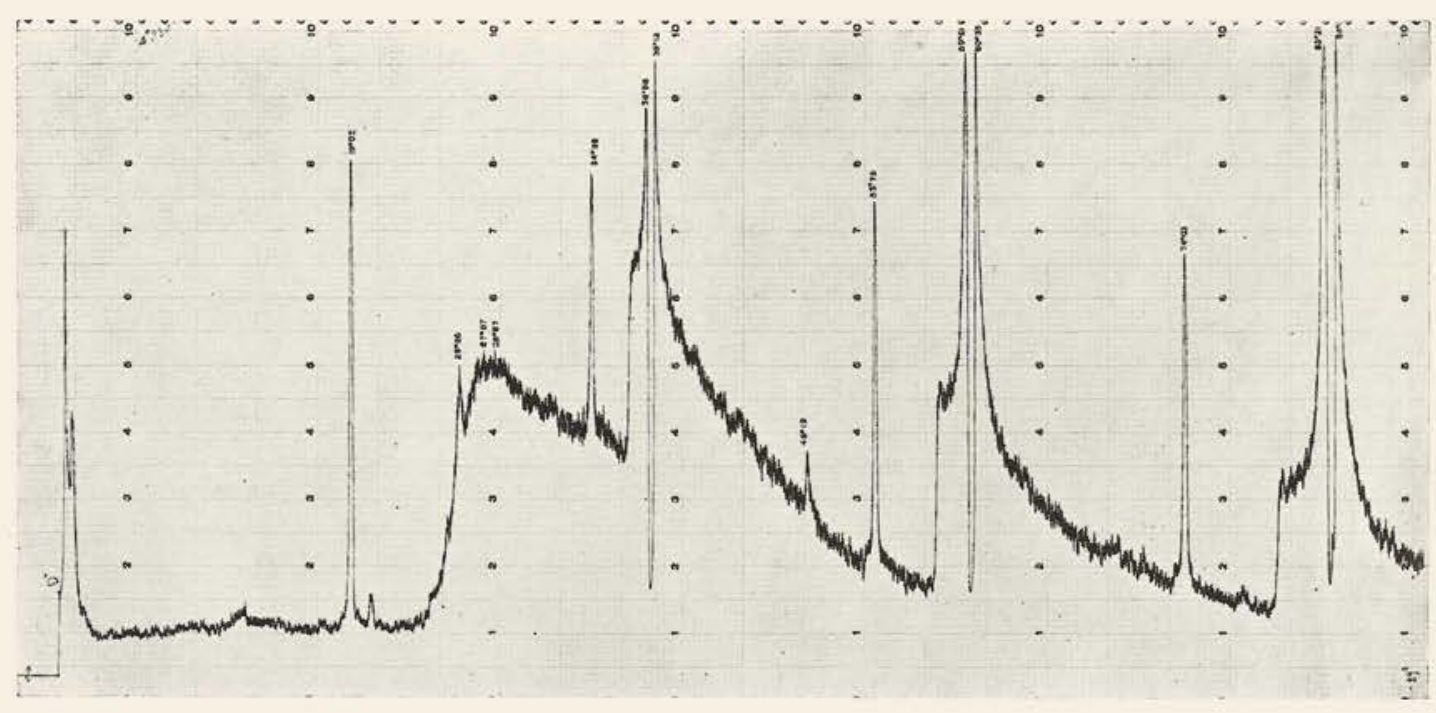

Diagrama de las caras de la base.

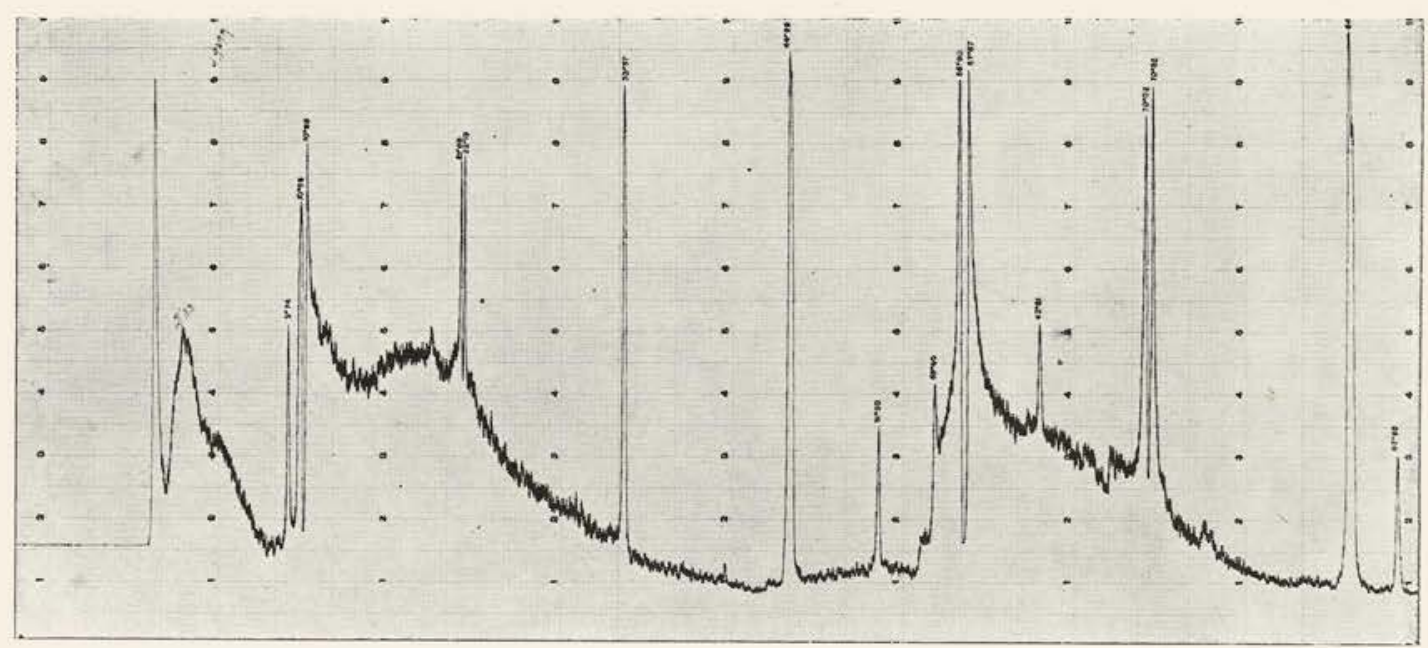

Diagrama de las earas laterales. 


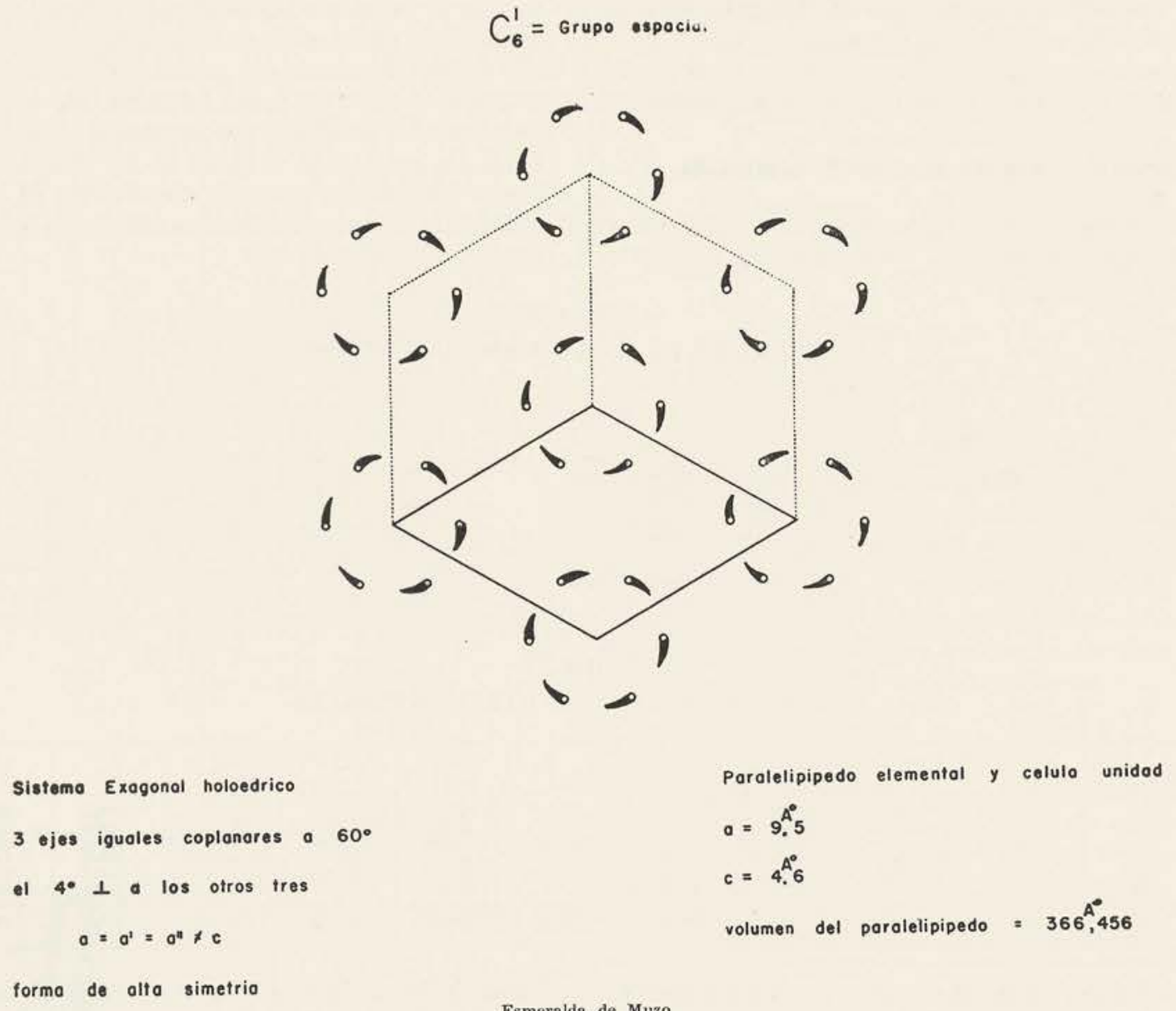

Esmeralda de Muzo

\section{Estructura de la célula unidad.}

Conocidas las dimensiones y el número de moléculas, es necesario fijar la posición de los átomos dentro del paralelipípedo elemental. No existen reglas para esto, pero se puede por medio de una serie de tanteos teniendo en cuenta las propiedades químicas y físicas, la capacidad de saturación de los átomos, el radio de estos, la simetría, y consultando las tablas de los espacio-grupos posibles de la forma cristalina seleccionada, llegar a establecer el edificio atómico con ex乏ctitud.

Para el Berilo, Bragg había seleccionado el espacio-grupo $\mathrm{D}_{6 \mathrm{~h}}^{2}$ o C /mec pero para la esmeralda, teniendo en cuenta la relación de los ejes cristalográficos medidos por los rayos $\mathrm{X}$, y conocido el contenido de la célula unidad, el espacio-grupo de la esmeralda de Muzo no puede ser sino $\mathrm{D}_{6 \mathrm{~h}}^{1}$ o $\mathrm{C}_{6} / \mathrm{mmm}$. por existir las reflexiones de los planos 001 y 100 tanto en el diagrama del polvo como en los de las caras de los monocristales.

La comprobación de la estructura por la comparación de las intensidades calculada y observada, no es posible en la esmeralda, debido al gran número de átomos dentro de la célula unidad (29 átomos). Las dos primeras líneas del diagrama del polvo dan resultados aceptables, pero las demás no son concordantes, y es especialmente notoria la diferencia de intensidades que muestran las mismas líneas de difracción en el polvo y en los cristales, lo cual, muy posiblemente se deba a las interferencias que necesariamente deben presentarse entre los múltiples cristalitos del polvo.

Prueba la posición de los átomos de Silicio en el plano de la base de la célula elemental, la magnitud deducida para el eje $c$, pues la reflexión debe originarse en ella. Los átomos de aluminio situados sobre los ejes ternarios de rotación a la mitad de distancia del plano de la base, lo mismo que los de berilium, situados a la misma altura en los ejes binarios, producen las interferencias por ocupar posiciones centradas en el retículo correspondiente. Los átomos de oxígeno forman un núcleo alrededor de cada átomo de silicio y cambian valencias con los metales aluminio y berilium, pues el grupo $\stackrel{0}{S}_{\delta_{1}^{\prime}}=0$ situado en cada uno de los vértices de un exágono regular deja las valencias libres para saturar los metales. 


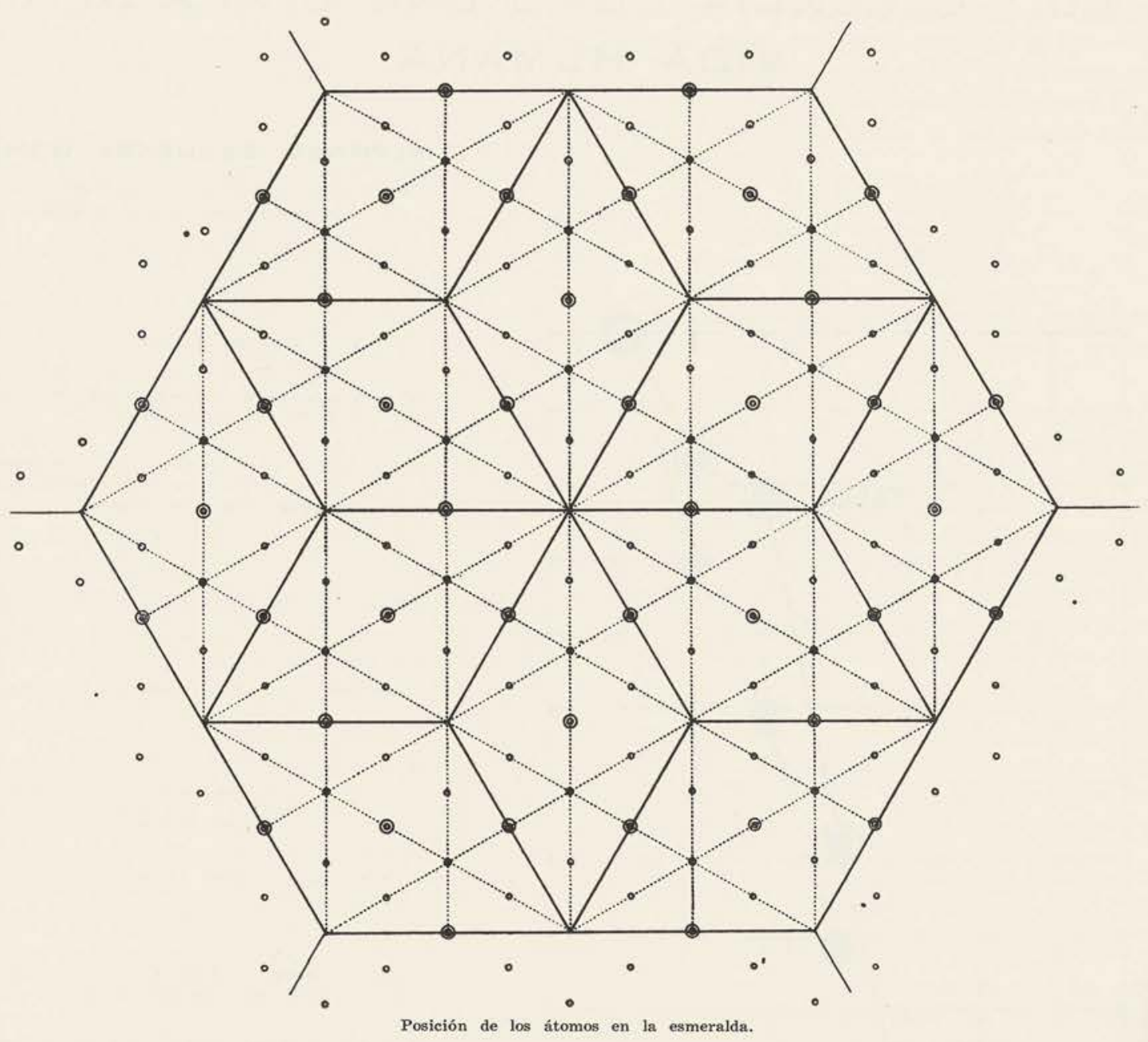

Las distancias entre los átomos cuadran con las magnitudes del retículo y los radios atómicos. En cualquier sentido que se tomen dentro de la célula se observa que ninguna de las órbitas atómicas se cruzan entre sí, y eléctricamente el prisma exagonal elemental, formado por los tres prismas romboidales resulta teóricamente neutro.
Por todo lo anteriormente expuesto, el espacio-grupo definitivo debe ser el de la figura anterior, con lo cual no solamente queda definida la gema, sino explicadas las dos formas comunes en la esmeralda de Muzo: la dextrorsum y la sinextrorsum, puesto que el eje de rotación puede existir en uno $u$ otro sentido. 